\title{
PROBING THE LOCAL BUBBLE WITH DIFFUSE INTERSTELLAR BANDS. III. THE NORTHERN HEMISPHERE DATA AND CATALOG
}

\author{
Amin Farhang ${ }^{1,2}$, Habib G. Khosroshahi ${ }^{1}$, Atefeh JAVAdi ${ }^{1}$, and JaCco Th. VAN LoON ${ }^{3}$ \\ ${ }^{1}$ School of Astronomy, Institute for Research in Fundamental Sciences (IPM), P.O. Box 19395-5746, Tehran, Iran; a.farhang@ipm.ir \\ ${ }^{2}$ Department of Physics, Sharif University of Technology, P.O. Box 11365-9161, Tehran, Iran \\ ${ }^{3}$ Astrophysics Group, Lennard-Jones Laboratories, Keele University, Staffordshire ST5 5BG, UK \\ Received 2014 November 11; accepted 2014 November 25; published 2015 February 10
}

\begin{abstract}
We present new high signal-to-noise ratio $(\mathrm{S} / \mathrm{N})$ observations of the diffuse interstellar bands (DIBs) in the Local Bubble and its surroundings. We observed 432 sightlines and obtain the equivalent widths of the $\lambda 5780$ and $\lambda 5797 \AA$ DIBs up to a distance of $\sim 200 \mathrm{pc}$. All of the observations were carried out using the Intermediate Dispersion Spectrograph on the $2.5 \mathrm{~m}$ Isaac Newton Telescope, during three years, to reach a minimum $\mathrm{S} / \mathrm{N}$ of $\sim 2000$. All of the $\lambda 5780$ and $\lambda 5797$ absorptions are presented in this paper and we tabulate the observed values of the interstellar parameters, $\lambda 5780, \lambda 5797, \mathrm{Na}_{\mathrm{ID}}$, and $\mathrm{Na}_{\mathrm{ID}}$, including the uncertainties.
\end{abstract}

Key words: ISM: abundances - ISM: bubbles - ISM: clouds - ISM: lines and bands - stars: atmospheres

Supporting material: extended figure, machine-readable table

\section{INTRODUCTION}

The Sun is located within a hot bubble (known as the Local Bubble) with a low neutral gas density of $n(\mathrm{H}) \sim$ $0.01 \mathrm{~cm}^{-3}$ (Bohlin 1975; Weaver et al. 1977) and a purportedly hot temperature $\left(T \sim 10^{6} \mathrm{~K}\right.$, but see Welsh \& Shelton (2009)), estimated based on the distribution of diffuse soft X-ray background emission (Snowden et al. 1998). According to recent observations of neutral gases (Na I), ionized atoms (Ca II), and dust grains, the Local Bubble is depleted of these common species (Vergely et al. 2001; Lallement et al. 2003; Welsh et al. 2010). Observations of nearby hot white dwarfs within the Local Bubble failed to detect an acceptable O vi absorption level (Oegerle et al. 2000). In addition, other observations of nearby white dwarfs and hot stars also failed to detect acceptable levels of highly ionized gas, such as C IV and Si IV (Bertin et al. 1995; Holberg et al. 1999), within the Local Bubble.

Three-dimensional (3D) gas maps around the Local Bubble reveal that this cavity has a chimney-like structure extending to a distance of $\sim 80 \mathrm{pc}$ in the Galactic Plane and up to hundreds of parsecs into the Halo (Vergely et al. 2001; Lallement et al. 2003; Welsh et al. 2010). Also, the diffuse X-ray background emission of the Local Bubble and its neighboring cavities has been studied to find a relation between the shape of the cavities and the soft X-ray data (Puspitarini et al. 2014). While the origin of the Local Bubble is still debated, the most plausible model is that the Local Bubble was created as the result of a number of successive supernovae in the nearby Sco-Cen association (Smith et al. 2001).

In recent years, about $\sim 500$ narrow-to-broad interstellar absorption features have been observed, which are known as diffuse interstellar bands (DIBs; Herbig 1995). The carriers of these heavy molecules are unknown, however, the DIB candidates could be among an infinite number of large carbonbased "organic" molecules (Sarre 2006). DIBs are used to study different conditions of the interstellar medium (ISM); for instance, some outstanding DIBs in the optical waveband are empirically known to trace the neutral phase of the ISM. Also, DIBs are well correlated with the color excess $E_{B-V}$, the neutral hydrogen, and the Na I column densities (Herbig 1993; Friedman et al. 2011).
DIBs react to the strength of the UV field of the local environment (Cox et al. 2006) and the DIB strength varies as a function of UV radiation toward different sightlines (Vos et al. 2011). Therefore, specific groups of UV-resistant molecules, such as polycyclic aromatic hydrocarbons, fullerenes, and carbon chains are commonly the more acceptable candidates for DIB carriers (Herbig 1995). DIB carriers have been ubiquitously detected everywhere, for instance, DIBs have been observed in M31 and M33 (Cordiner et al. 2008a, 2008b), Magellanic Clouds (van Loon et al. 2013), and beyond the Milky Way in supernova host galaxies (Cox \& Patat 2008).

Since the temperature of the Local Bubble is purportedly high, typical atoms and molecules could not survive in such an environment. In addition, as mentioned above, any attempts to detect other highly ionized atoms, or to search for an acceptable level of EUV emission, have failed. Therefore, we study the Local Bubble and its surroundings through DIBs in a survey over a three years period. We present the sightlines, spectrums, fitted profiles, and equivalent widths (EWs) for the northern hemisphere survey.

\section{OBSERVATIONS}

All of the observations have been obtained with the Intermediate Dispersion Spectrograph (IDS) at the $2.5 \mathrm{~m}$ Isaac Newton Telescope at the Roque de Los Muchachos in La Palma. IDS employs a long-slit spectrograph with two CCDs: the EEV10 CCD, sensitive in blue, and RED+2, which is sensitive in the red, and both CCDs have $4096 \times 2048$ pixels. The spatial scale for EEV10 is 0'.4 and for RED+2 is $0{ }^{\prime \prime} 44$ pixel $^{-1}$, and the full unvignetted slit length is 3.3 .

For our observations, we have used the $235 \mathrm{~mm}$ camera and H1800V IDS grating for an effective resolution of $0.31 \AA$ pixel $^{-1}$. H1800V was chosen since it provides a high spectral resolution that is well matched to the typical width of DIBs. Also, we have chosen $5800 \AA$ as the central wavelength. A 1'.1 slit yielded spectra in the 5750-6040 ̊ region at spectral resolutions of $R \equiv \lambda / \Delta \lambda \sim 2000$ (or a velocity resolution of $\Delta v=150 \mathrm{~km} \mathrm{~s}^{-1}$ ). The DIB detection requires a high signalto-noise ratio $(\mathrm{S} / \mathrm{N})$ of at least 100 , but for the detection of very weak absorptions, similar to the one detected by Cordiner 

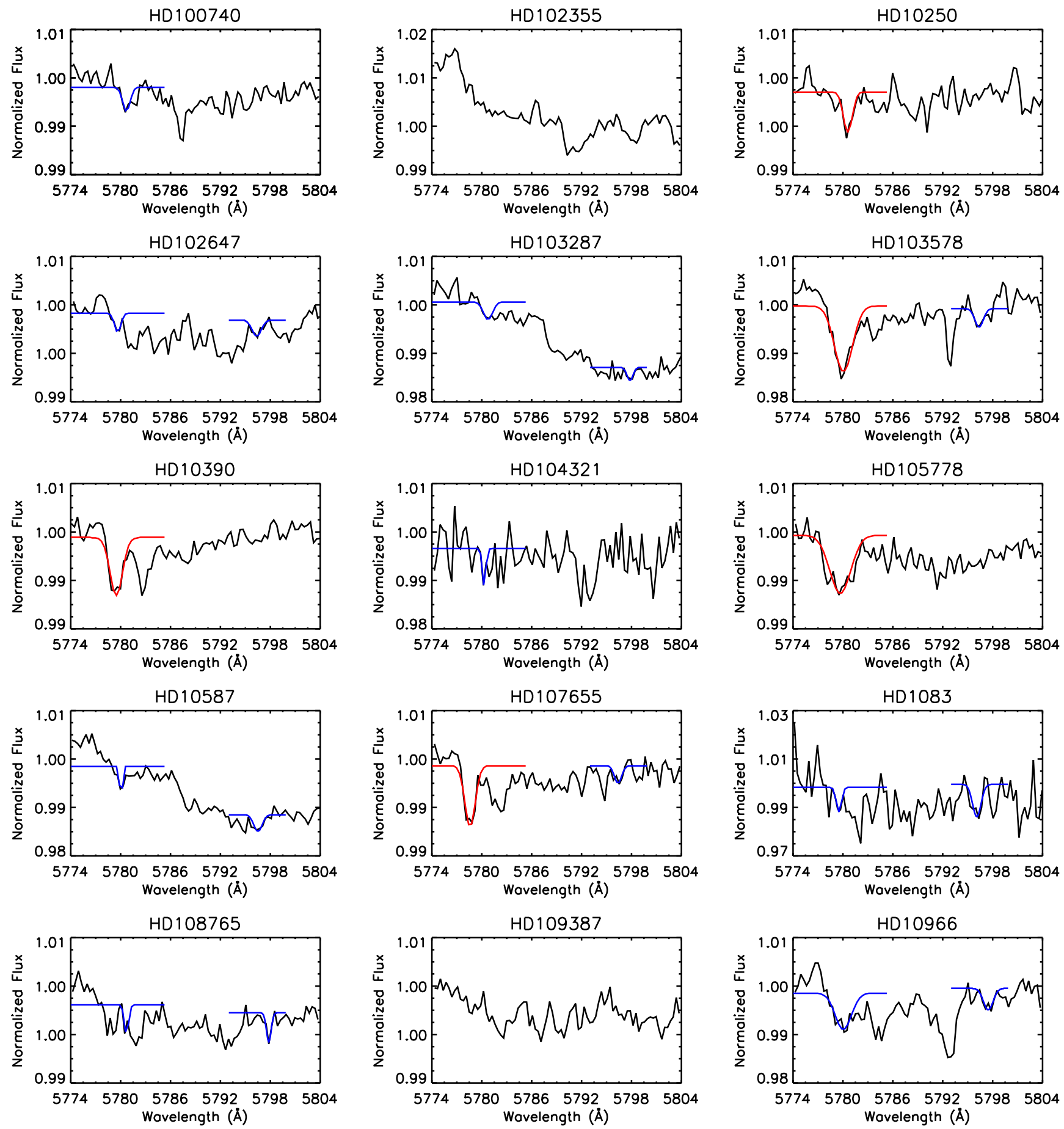

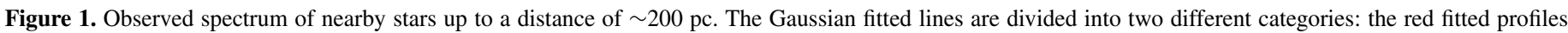
show acceptable DIBs and the blue lines represents the uncertain DIB features.

(An extended version of this figure is available.)

(2006) toward the nearby star $\mu^{1}$ Cru with a $\lambda 5780$ DIB EW of $4 \mathrm{~m} \AA$, we need an $\mathrm{S} / \mathrm{N}$ of at least 2000 . The seeing varied during these observing nights from 1 .' 1 to $1^{\prime \prime} .9$.

To achieve high-quality data, we obtained more than 60 flat field frames with a quartz lamp (exposure times of 13-15 s), 15 arc frames $(\mathrm{CuAr}+\mathrm{CuNe})$ for wavelength calibration, and a large number of bias frames every night. In order to achieve a minimum $\mathrm{S} / \mathrm{N} \sim 2000$, we exposed each target 9-25 times, depending on their apparent magnitudes (see below). The data were processed using the CCDRED data reduction package and the spectrum were extracted using the KPNOSLIT package.
We have selected bright stars up to a distance of 200 pc. Targets were selected from the 3D Na I D lines survey of Welsh et al. (2010), all with well-known distances from the Hipparcos satellite (Perryman et al. 1997). Since the saturation level of the IDS detector was $\sim 64,000$ counts, a small number of targets were rejected due to their extreme brightness $(V<1.8 \mathrm{mag}$ ). To increase the observing run efficiency, targets with $V>7.2 \mathrm{mag}$ were also rejected from our target list to avoid very long integration times. Also, to maximize the uniformity of the survey, in addition to the observation of hot stars ( $\mathrm{O}, \mathrm{B}$ types), we also observed some cooler stars (A, F, G, and K). 
Table 1

The EW Measurements of $\lambda 5780, \lambda 5797$, and Na I Absorptions in the Northern Hemisphere up to a Distance of $\sim 200 \mathrm{pc}$

\begin{tabular}{|c|c|c|c|c|c|c|c|c|c|c|c|c|c|c|}
\hline Name & Glon & Glat & Dis & $\lambda 5780$ & Err $\lambda 5780$ & $\lambda 5797$ & Err $\lambda 5797$ & $\mathrm{NaI}_{2}$ & Err $\mathrm{Na}_{\mathrm{I}} \mathrm{D}_{2}$ & $\mathrm{Na} \mathrm{I}_{1}$ & Err Na I D & f1 & $\mathrm{f} 2$ & $\mathrm{f3}$ \\
\hline HD 10250 & 127.2 & 8.2 & 86 & 7.47 & 1.95 & 0.00 & 0.00 & 0.00 & 0.00 & 0.00 & 0.00 & 1 & 0 & 0 \\
\hline HD 10390 & 134.5 & -26.5 & 79 & 16.30 & 2.97 & 0.00 & 0.00 & 0.00 & 0.00 & 0.00 & 0.00 & 1 & 0 & 0 \\
\hline HD 10587 & 130.2 & -5.0 & 172 & 3.33 & 0.93 & 4.64 & 1.25 & 0.00 & 0.00 & 0.00 & 0.00 & 0 & 0 & 0 \\
\hline HD 1083 & 113.1 & -34.9 & 126 & 7.75 & 2.74 & 16.42 & 3.14 & 0.00 & 0.00 & 0.00 & 0.00 & 0 & 0 & 0 \\
\hline HD 11291 & 132.6 & -10.9 & 151 & 0.00 & 0.00 & 0.00 & 0.00 & 46.34 & 12.36 & 69.17 & 11.85 & 1 & 1 & 1 \\
\hline HD 11335 & 132.6 & -10.2 & 152 & 3.53 & 2.10 & 0.00 & 0.00 & 65.42 & 11.72 & 56.51 & 10.97 & 0 & 0 & 1 \\
\hline HD 11415 & 129.8 & 1.6 & 136 & 22.59 & 3.58 & 1.63 & 0.67 & 55.26 & 9.18 & 33.96 & 8.52 & 1 & 0 & 1 \\
\hline HD 11946 & 130.2 & 2.7 & 79 & 6.46 & 1.70 & 0.00 & 0.00 & 0.00 & 0.00 & 0.00 & 0.00 & 1 & 1 & 0 \\
\hline HD 12216 & 128.4 & 10.3 & 50 & 16.73 & 2.81 & 2.17 & 0.76 & 161.15 & 16.00 & 251.97 & 16.19 & 1 & 0 & 0 \\
\hline HD 1280 & 115.6 & -23.7 & 78 & 27.03 & 4.63 & 6.95 & 1.15 & 224.64 & 18.10 & 275.48 & 18.60 & 1 & 1 & 0 \\
\hline
\end{tabular}

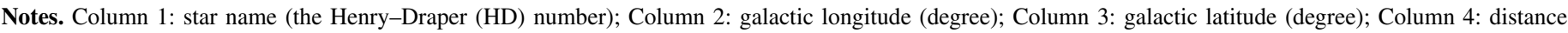

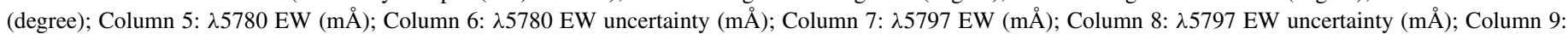

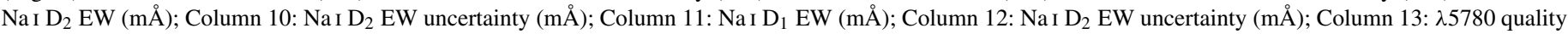

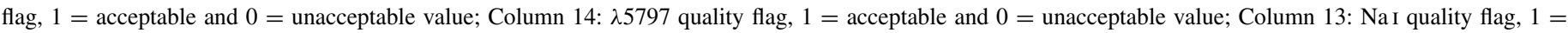
acceptable and $0=$ unacceptable value.

(This table is available in its entirety in machine-readable form.)

\section{DATA ANALYSIS}

In DIB studies, the shapes of spectral profiles remain uncertain since the carriers are unidentified. In addition, blending by other chemical species and the possibility for blending with features from higher rotational levels of the same species (Friedman et al. 2011) make DIB studies challenging. The EW is defined as

$$
W=\int \frac{I_{0}(\lambda)-I(\lambda)}{I_{0}(\lambda)} d \lambda,
$$

where $I_{0}$ and $I_{\lambda}$ are the fluxes of the continuum and the spectral line, respectively. With a Gaussian function, one can approximate the $\lambda 5780$ and $\lambda 5797$ line absorptions (e.g., van Loon et al. 2009). Therefore, we determine the line width in terms of the $\sigma$ value of the Gaussian distribution, and then calculate the FWHM as FWHM $=2(2 \ln 2)^{1 / 2} \sigma=2.355 \sigma$. Accordingly, to produce a normalized spectrum, we performed continuum fitting to the observed spectra by fitting a ninth-order Legendre polynomial. Some of our Gaussian fitting to normalized spectra are plotted in Figure 1.

To compute the statistical uncertainty for each observed DIB, we compute the standard deviation of the residuals of the Gaussian fitting, summed in quadrature and weighted by the Gaussian fit (van Loon et al. 2009; Vos et al. 2011). In DIB studies, the main source of error in the EWs is the uncertainty in determining the real position of the continuum (systematic error), and therefore the statistical error is always underestimated. To compute the systematic error, we fit three different continuum lines to the local absorption region with $\pm 12 \AA$ A range around the central DIB wavelengths (linear fit to the continuum, quadratic fit to the continuum, and fit to DIB with linear continuum (Kos \& Zwitter 2013)). Accordingly, we set the intersection points of the DIB absorption and the continuum level (Krełowski \& Sneden 1993). Therefore, the systematic uncertainty is determined by the difference between the highest and lowest EW values among these three EWs.

To extract the ISM absorption of cold stars, it is necessary to simulate the spectrum of cold stars. To construct the synthetic spectrum of cold stars, we calculate atmosphere models using the ATLAS9 code (Kurucz 1992). We use grids of ATLAS9 model atmospheres from Castelli \& Kurucz $(2003)^{4}$ with new

\footnotetext{
4 http://wwwuser.oats.inaf.it/castelli/
}

opacity distribution functions for several metallicities (Castelli et al. 1997). We obtained the effective temperature of the observed star and the surface gravity $\log g$ from Cayrel et al. (1996), Varenne \& Monier (1999), Reddy et al. (2003), Prugniel et al. (2007, 2011), Huang et al. (2010) and Koleva \& Vazdekis (2012). Also, we determined the target metallicity from Heacox (1979), Abt et al. (2002), Royer et al. (2002, 2007) and Schróder et al. (2009). After modeling the stellar atmosphere, we generated the synthetic stellar spectrum with the Linux port of the SYNTHE suite codes (Sbordone et al. 2004; Sbordone 2005; Kurucz 2005). Atomic and molecular data were taken from the database on the Kurucz website ${ }^{5}$ (Kurucz 2005). Also, to consider the broadening caused by the rotational velocity, we use the rotational velocity from Abt et al. (2002); Royer et al. (2002, 2007) and Schróder et al. (2009). For those stars for which the rotational velocity was not reported, we use $v \sin i=15-20 \mathrm{~km} \mathrm{~s}^{-1}$. In our calculations, we included all of the atomic and molecular lines with empirically determined atomic constants as well as all of the diatomic molecular lines ( $\mathrm{CH}, \mathrm{NH}, \mathrm{CN}, \mathrm{MgH}, \mathrm{SiH}, \mathrm{SiO}, \mathrm{H}_{2}, \mathrm{C}_{2}$, and $\mathrm{CO}$ ), except for the TiO molecule.

The A-type stars have two main sources of contamination Fe II (at $5780.13 \AA$ and $5780.37 \AA$ ) and Fe II (at $5783.63 \AA$ ) at the $\lambda 5780$ position. Also, there is no source of contamination at $\lambda 5797$. To correct these contaminations for each A-type star for which the metallicity has not been reported, we produce all of the possible synthetic spectra and compare them with the observed spectra. The surface gravity of A-type stars varies from 3.5 to 4.2 (Gray 1992), and therefore we choose a constant $\log g=4$ in all of our atmosphere models. From the A0 to A9 spectral types, according to the Theodossiou \& Danezis (1991) report, we select a constant temperature for each I, II-III, and IV-V luminosity class. Also, the A-type stars have different metallicities from 0 to -2 (Beers et al. 2001). Accordingly, for a given A-type subdivision (e.g., A0) and luminosity class (e.g., IV), we produce three different atmosphere models with $[\mathrm{Fe} / \mathrm{H}]=0,-0.5,-1.5$, and compare with the observed spectrum to choose the best model. Also, we consider the effect of rotational velocity convolved with instrument dispersion. However, the rotational velocities of our observed A-type

\footnotetext{
5 http://kurucz.harvard.edu
} 
stars are very high $\left(\sim 200 \mathrm{~km} \mathrm{~s}^{-1}\right)$ (Hoffleit \& Warren 1995); thus, when convolved with our instrument dispersion, the absorption lines are widened. Therefore, the impact of their stellar features on DIB absorption will be limited. Then, we subtract the synthetic spectrum from the observed absorption (containing both stellar and interstellar absorption features). The obtained residual predominantly consists of interstellar absorption (Montes et al. 1995a, 1995b). Then, by fitting a Gaussian function to this residual, the EW of the DIB will be obtained.

The spectrum shape of cool F-, G-, and K-type stars is similar to a two-pronged fork. These absorptions are caused by the presence of Fe II, MnI, Si I (all near $5780.1 \AA$ ), and CrI (5781.1 $\AA$ ). Therefore, to produce the synthetic spectrum of these cool stars, we consider a Gaussian profile for $\lambda 5780$ absorption lines:

$$
D(\lambda)=a \exp \left(-\frac{(\lambda-b)^{2}}{2 \sigma}\right)+c
$$

After confirming the average $\lambda 5780$ DIB profile with an iterative procedure, according to Equation (2), we change $a$ (peak intensity), $b$ (peak center), and $\sigma$ (peak with) for each wavelength $(\lambda)$ to produce a new spectrum. Then, we add this spectrum to the corresponding synthetic spectrum, and in each iteration, according to Equation (3), we calculate the difference of the reproduced spectrum with the real observed absorption $\left(\chi^{2}\right)$. Eventually, the best DIB profile estimation is that with the smallest $\chi^{2}$ value:

$$
\chi^{2}=\sum_{i=1}^{N}\left(\frac{\left(F\left(\lambda_{i}\right)-D\left(\lambda_{i}\right)\right)^{2}}{F_{\mathrm{err}}\left(\lambda_{i}\right)^{2}}\right)
$$

In our plots, we show the observed spectra and their best Gaussian fit to the $\lambda 5780$ and $\lambda 5797$ features (Figure 1). The red fits are those with acceptable DIB absorption and the blue fits are those for which we are not confident about their DIB character. Also, the tables show the $\lambda 5780 \mathrm{EW}$ and its uncertainty, the $\lambda 5797 \mathrm{EW}$ and its uncertainties, the $\mathrm{Na} \mathrm{I}_{2}$ and $\mathrm{Na} \mathrm{I}_{1}$ and their uncertainties, and the target distances (small sample of measurements are listed in Table 1). The last three columns are the $\lambda 5780, \lambda 5797$, and $\mathrm{NaI}$ flags, where 1 indicates that the quality of measurements are acceptable and 0 means that they are unacceptable values.

We wish to thank the Iranian National Observatory (INO) and School of Astronomy at IPM for facilitating and supporting this project. The observing time allocated to this project was provided by the INO. We also wish to thank the ING staff for their support. Some of the research visits related to this project have been supported by the Royal Society International Exchange Scheme. Furthermore, we thank the anonymous referee for a thorough reading of the manuscript and useful comments and suggestions.

\section{REFERENCES}

Abt, H. A., Levato, H., \& Grosso, M. 2002, A\&A, 573, 359

Beers, T. C., Rossi, S., O'Donoghue, D., et al. 2001, MNRAS, 320, 451

Bertin, P., Vidal-Madjar, A., Lallement, R., Ferlet, R., \& Lemoine, M. 1995, A\&A, 302, 889

Bohlin, R. 1975, ApJ, 200, 402

Castelli, F., Gratton, R. G., \& Kurucz, R. L. 1997, A\&A, 318, 841

Castelli, F., \& Kurucz, R. L. 2003, in IAU Symp. 210, Modelling of Stellar Atmospheres, ed. N. Piskunov \& W. W. Weiss (Uppsala: Uppsala Univ.), 20

Cayrel de Strobel, G., Soubiran, C., Friel, E. D., Ralite, N., \& François, P. 1996, yCat, 3200, 0

Cordiner, M. A. 2006, PhD thesis, The Univ. Nottingham

Cordiner, M. A., Cox, N. L. J., Trundle, C., et al. 2008a, A\&A, 480, 13

Cordiner, M. A., Smith, K. T., Cox, N. L. J., et al. 2008b, A\&A, 492, 5

Cox, N. L. J., Cordiner, M. A., Cami, J., et al. 2006, A\&A, 447, 991

Cox, N. L. J., \& Patat, F. 2008, A\&A, 485, 9

Friedman, S. D., York, D. G., McCall, B. J., et al. 2011, ApJ, 727, 33

Gray, D. F. 1992, Cambridge Astrophysics Ser., vol. 20, The Observation and Analysis of Stellar Photospheres, (3rd ed.; Cambridge: Cambridge Univ. Press)

Heacox, W. D. 1979, ApJS, 41, 675

Herbig, G. H. 1993, ApJ, 407, 142

Herbig, G. H. 1995, ARA\&A, 33, 19

Hoffleit, D., \& Warren, W. H., Jr. 1995, yCat, 5050, 0

Holberg, J. B., Barstow, M. A., Bruhweiler, F. C., Hubeny, I., \& Green, E. M. 1999, ApJ, 517, 850

Huang, W., Gies, D. R., \& McSwain, M. V. 2010, ApJ, 722, 605

Koleva, M., \& Vazdekis, A. 2012, A\&A, 538, 143

Kos, J., \& Zwitter, T. 2013, ApJ, 774, 72

Krełowski, J., \& Sneden, C. 1993, PASP, 105, 1141

Kurucz, R. L. 1992, RMxAA, 23, 45

Kurucz, R. L. 2005, MmSAI, 8, 14

Lallement, R., Welsh, B. Y., Vergely, J. L., et al. 2003, A\&A, 411, 447

Montes, D., De Casto, E., Fernandes-Figueroa, M. J., \& Cornide, M. 1995a, A\&AS, 114, 187

Montes, D., De Casto, E., Fernandes-Figueroa, M. J., \& Cornide, M. 1995b, A\&AS, 109, 135

Oegerle, W. R., Tripp, T. M., Sembach, K. R., et al. 2000, ApJL, 538, L23

Perryman, M. A. C., Lindegren, L., Kovalevsky, J., et al. 1997, A\&A, 323, 49

Prugniel, P., Soubiran, C., Koleva, M., \& Le Borgne, D. 2007, yCat, 3251, 0

Prugniel, P., Vauglin, I., \& Koleva, M. 2011, A\&A, 531, A165

Puspitarini, L., Lallement, R., Vergely, J. L., \& Snowden, S. L. 2014, A\&A, 566, A13

Reddy, B. E., Tomkin, J., Lambert, D. L., \& Allende Prieto, C. 2003, MNRAS, 340, 304

Royer, F., Grenier, S., Baylac, M. O., Gómez, A. E., \& Zorec, J. 2002, A\&A, 393, 897

Royer, F., Zorec, J., \& Gómez, A. E. 2007, A\&A, 463, 671

Sarre, P. J. 2006, JMoSp, 238, 1

Sbordone, L. 2005, MmSAI, 8, 61

Sbordone, L., Bonifacio, P., Castelli, F., \& Kurucz, R. L. 2004, MSAIS, 5, 93

Schróder, C., Reiners, A., \& Schmitt, J. H. M. M. 2009, A\&A, 493, 1099

Smith, L. J., \& Gallagher, J. S. 2001, MNRAS, 326, 1027

Snowden, S. L., Egger, R., Finkbeiner, D., Freyberg, M., \& Plucinsky, P. 1998, ApJ, 493, 715

Theodossiou, E., \& Danezis, E. 1991, Ap\&SS, 183, 91

van Loon, J. Th., Smith, K. T., McDonald, I., et al. 2009, MNRAS, 399, 195

van Loon, J. Th., Bailey, M., Tatton, B. L., et al. 2013, A\&A, 550, 108

Varenne, O., \& Monier, R. 1999, A\&A, 351, 247

Vergely, J. L., Freire, F. R., Siebert, A., \& Valette, B. 2001, A\&A, 366, 1016

Vos, D. A. I., Cox, N. L. J., Kaper, L., Spaans, M., \& Ehrenfreund, P. 2011, A\&A, 533, A129

Weaver, R., McCray, R., Castor, J., Shapiro, P., \& Moore, R. 1977, ApJ, 218, 377

Welsh, B. Y., Lallement, R., Vergely, J. L., \& Raimond, S. 2010, A\&A, 510, A54

Welsh, B. Y., \& Shelton, R. L. 2009, Ap\&SS, 323, 1 